\title{
THE HILBERT SCHEME OF A PLANE CURVE SINGULARITY AND THE HOMFLY POLYNOMIAL OF ITS LINK
}

\author{
ALEXEI OBLOMKOV AND VIVEK SHENDE
}

\begin{abstract}
The intersection of a complex plane curve with a small three-sphere surrounding one of its singularities is a non-trivial link. The refined punctual Hilbert schemes of the singularity parameterize subschemes supported at the singular point of fixed length and whose defining ideals have a fixed number of generators. We conjecture that the generating function of Euler characteristics of refined punctual Hilbert schemes is the HOMFLY polynomial of the link. The conjecture is verified for irreducible singularities $y^{k}=x^{n}$, whose links are the $(k, n)$ torus knots, and for the singularity $y^{4}=x^{7}-x^{6}+4 x^{5} y+2 x^{3} y^{2}$, whose link is the $(2,13)$ cable of the trefoil.
\end{abstract}

\section{INTRODUCTION}

Let $C$ be an integral complex curve with at worst locally planar singularities. We write $C^{[l]}$ for its Hilbert scheme of points [G], the moduli space of closed subschemes of $C$ of dimension zero and length $l$. We are interested in the Euler characteristic 1 of these spaces. For notational convenience, we write $C^{[\star]}:=\coprod C^{[l]}$, and separate the components by the function $l: C^{[\star]} \rightarrow \mathbb{N}$ counting the number of points. In the case of a smooth curve $C_{\mathrm{sm}}$, not necessarily complete, the following formula is well known:

$$
\int_{C_{\mathrm{sm}}^{[\downarrow]}} q^{2 l} d \chi=\left(\frac{1}{1-q^{2}}\right)^{\chi\left(C_{\mathrm{sm}}\right)}
$$

For any point $p \in C$, denote by $C_{p}^{[l]}$ the closed subvariety of $C^{[l]}$ whose closed points parameterize subschemes of $C$ that are set-theoretically supported at $p$. We collect these into $C_{p}^{[\star]}=\amalg C_{p}^{[l]}$ which we equip with a function $l$ giving the length. Now let $p_{i}$ be the singular points of $C$, and take $C_{\mathrm{sm}}:=C \backslash \amalg p_{i}$. Stratifying $C^{[\star]}$ by the length supported at the singular points, we find:

$$
\int_{C^{[\star]}} q^{2 l} d \chi=\int_{C_{\mathrm{sm}}} q^{2 l} d \chi \times \prod_{i} \int_{C_{P_{i}}^{[\downarrow]}} q^{2 l} d \chi=\left(\frac{1}{1-q^{2}}\right)^{\chi\left(C_{\mathrm{sm}}\right)} \prod_{i} \int_{C_{P_{i}}^{[\star]}} q^{2 l} d \chi
$$

The contributions from the singularities can be rather complicated. We will describe them conjecturally in terms of the topology of $C$ near its singular points. Embed $C$ in a surface. Intersecting $C$ with a small 3-sphere around $p \in C$ yields the link of $C$ at $p$ : a collection of oriented circles in $S^{3}$, one for each analytic branch of $C$ at $p$. For example, the curve $y^{k}=x^{n}$ intersects a sphere around the origin in the right-handed $(k, n)$ torus link. Milnor $[\overline{\mathrm{M}}]$ has studied links of hypersurface

\footnotetext{
${ }^{1}$ For a topological space $Y$, we write $\chi(Y)=\sum(-1)^{i} \operatorname{dim} \mathrm{H}^{i}(Y, \mathbb{Q})$; for specificity, we take singular cohomology. When $Y$ is a complex algebraic variety, this is equal to the analogous $\chi_{c}(Y)$ formed from compactly supported cohomology. If $Z$ a closed subvariety of $Y$, then $\chi(Y \backslash Z)=\chi(Y)-\chi(Z)$. If $E \rightarrow B$ is a topologically locally trivial fibration with fibre $F$, then $\chi(E)=\chi(F) \chi(B)$. In particular, if $Y$ admits the action of an algebraic torus $T=\left(\mathbb{C}^{*}\right)^{n}$, then $\chi(Y)=\# Y^{T}$. We use integral notation for weighted Euler characteristics: if $\phi: Y \rightarrow A$ is a constructible function taking values in an abelian group, then $\int_{Y} \phi d \chi:=\sum a \cdot \chi\left(\phi^{-1}(a)\right)$.
} 
singularities, and shows in particular that the complement in the sphere of any such link is a smooth fibre bundle over the circle. Eisenbud and Neumann $[\mathrm{EN}]$ describe how to pass from the combinatorics of the Puiseux data of the singularity to a presentation of the link. Campillo, Delgado, and Gusein-Zade have shown that the Alexander polynomial of the link carries data equivalent to the Hilbert series of a certain filtration on the ring of functions on the singularity [CDG].

Denote by $\overline{\mathbf{P}}(L)$ the HOMFLY polynomial of an oriented link $L \subset S^{3}$. It is an element of $\mathbb{Z}\left[a^{ \pm 1},\left(q-q^{-1}\right)^{ \pm 1}\right]$, and may be computed from the relations

$$
\begin{aligned}
a \overline{\mathbf{P}}\left(\lambda^{\curlywedge}\right)-a^{-1} \overline{\mathbf{P}}(\nwarrow) & =\left(q-q^{-1}\right) \overline{\mathbf{P}}()() \\
a-a^{-1} & =\left(q-q^{-1}\right) \overline{\mathbf{P}}(\bigcirc)
\end{aligned}
$$

The terms of Equation (2) should be interpreted as the HOMFLY polynomials of three link diagrams which are identical away from a small neighborhood, and are as depicted within it. It is not obvious that these relations define a function on diagrams, let alone knots, but it is true [HOMFLY]. It is often convenient to adopt the alternative normalization $\mathbf{P}(L):=\overline{\mathbf{P}}(L) / \overline{\mathbf{P}}(\bigcirc)$. The topological meaning of the HOMFLY polynomial is unknown.

Conjecture 1. Let $C$ be a curve in a smooth surface, $C$ itself smooth away from points $p_{i}$. Let $L_{i}$ be the link of $C$ at $p_{i}$, and let $\mu_{i}$ be the Milnor number of the singularity at $p_{i}$. Then,

$$
\int_{C[\star]} q^{2 l} d \chi=\left(1-q^{2}\right)^{-\chi(C)} \prod_{i}\left[(q / a)^{\mu_{i}} \mathbf{P}\left(L_{i}\right)\right]_{a=0}
$$

Aside. We pause to mention the role of the series on the left hand side of the conjecture in the curve-counting theory of Pandharipande and Thomas. If $\tilde{g}$ and $g$ are the geometric and arithmetic genera of $C$, then there are integers $n_{h}$ for $\tilde{g} \leq h \leq g$ such that [PT3]

$$
\int_{C[\star]} q^{2 l} d \chi=q^{2 g-2} \sum_{h=\tilde{g}}^{g} n_{h}(C)\left(q^{-1}-q\right)^{2 h-2}
$$

The second author has shown $[\mathbf{S}]$ that these integers are positive, and record the degree of the subvariety of the versal deformation of $C$ parameterizing deformations with $g-h$ nodes. In the simplest cases, for instance if $C$ is a curve in a Fano surface of an irreducible homology class $\gamma$, the $n_{h}(C)$ give the local contribution of $C$ to the Gopakumar-Vafa [GV] invariant measuring the number of genus $h$ curves of class $\gamma$ in the local Calabi-Yau formed by the canonical bundle of the surface. We are unaware of any physical interpretation of such local contributions: roughly speaking, the Gopakumar-Vafa numbers arise from identifying a certain space of BPS states in M-theory compactified on the Calabi-Yau of interest with the cohomology of the moduli space of rank one sheaves supported on any curve of class $\gamma$.

There is also a connection to quantum field theory on the other side of the conjecture: Witten [W] has explained that the HOMFLY polynomial may be understood as collecting, over $k$ and $N$, the expectation value of the Wilson loop around the link in the $\mathrm{SU}(N)$ Chern-Simons theory on the three-sphere at level $k$. One might hope to give a "physics proof" of the above conjecture via a theory which, on the one hand gives rise to local Gopakumar-Vafa numbers, and, on the other, specializes near any point of $C$ to Chern-Simons theory on the bounding 3-sphere.

We turn to the question of how the HOMFLY polynomial, rather than merely its $a \rightarrow 0$ limit, may be recovered from the Hilbert scheme. We define an incidence variety:

$$
C_{p}^{[l]} \times C_{p}^{[l+m]} \supset C_{p}^{[l, l+m]}:=\left\{(I, J) \mid I \supset J \supset I \mathcal{O}_{C}(-p)\right\}
$$


Conjecture 2. Let $p \in C$ be a point on a locally planar curve, let $L_{C, p}$ be the link of $C$ at $p$, and let $\mu$ be the Milnor number of the singularity at $p$. Then,

$$
\overline{\mathbf{P}}\left(L_{C, p}\right)=(a / q)^{\mu-1} \sum_{l, m} q^{2 l}\left(-a^{2}\right)^{m} \chi\left(C_{p}^{[l, l+m]}\right)
$$

We may integrate out the incidence variety. By Nakayama's lemma, the possible $J$ for given $I$ are parameterized by a Grassmannian inside $I / I \mathcal{O}_{C}(-p)=I \otimes \mathbb{C}_{p}$. Define

$$
\begin{aligned}
m: C_{p}^{[\star]} & \rightarrow \mathbb{N} \\
I & \mapsto \operatorname{dim} I \otimes \mathbb{C}_{p}
\end{aligned}
$$

Equivalently, $m(I)$ is the minimal number of generators of the ideal $I \otimes \hat{\mathcal{O}}_{C, p}$. The contribution of $I$ to the RHS of Conjecture 2 is $\left(1-a^{2}\right)^{m(I)}$, so we have the following equivalent formulation (which we have given in terms of the normalized HOMFLY polynomial):

Conjecture 2'. Let $p \in C$ be a point on a locally planar curve, let $L_{C, p}$ be the link of $C$ at $p$, and let $\mu$ be the Milnor number of the singularity at $p$. Then,

$$
\mathbf{P}\left(L_{C, p}\right)=(a / q)^{\mu}\left(1-q^{2}\right) \int_{C_{p}[*]} q^{2 l}\left(1-a^{2}\right)^{m-1} d \chi
$$

Conjecture 2 implies Conjecture 1 by a stratification argument. The remainder of the article presents evidence for the conjectures, which we briefly itemize below.

- Setting $a=-1$ in Conjecture 2 leaves the formula

$$
\nabla\left(L_{C, p}\right)=(-q)^{-\mu}\left(1-q^{2}\right) \int_{\mathcal{O}_{C, p} / \mathcal{O}_{C, p}^{*}} q^{2 l} d \chi
$$

where $\nabla$ is the Alexander polynomial in a suitable normalization, and $\mathcal{O}_{C, p} / \mathcal{O}_{C, p}^{*}$ parameterizes functions up to multiplication by invertible functions, or in other words, ideals with one generator. After some technical rearrangements, this formula follows from a theorem of Campillo, Delgado, and Gusein-Zade. Details appear in Section 3 ,

- The skein relation exhibits the invariance the HOMFLY polynomial under the transformation $q \rightarrow-q^{-1}$; in fact, $\mathbf{P} \in \mathbb{Z}\left[\left(q-q^{-1}\right)^{ \pm 1}, a^{ \pm 1}\right]$. It is not evident from their expressions that our integrals enjoy the same property; nonetheless this was verified by Pandharipande and Thomas for the integral in Conjecture 1, and we explain in Section 4 how to extend their methods to the integral in Conjecture 2.

- Singularities of the form $y^{k}=x^{n}$ carry a torus action which lifts to the Hilbert scheme. In the case $\operatorname{gcd}(k, n)=1$, the fixed points are isolated, and we count them in Section 5 to calculate the integral in Conjecture 2. Jones has calculated the HOMFLY polynomial of the corresponding $(k, n)$-torus knot, and the formulae match.

- Section 6 describes, in the case of unibranch singularities, a stratification of the Hilbert scheme of points via the semigroup of the singularity. This is closely related to Piontkowski's work on computing the cohomology of compactified Jacobians [Pi]. In Section 7 we compute explicitly the strata of the Hilbert scheme of the singularity with complete local ring $\mathbb{C}\left[\left[t^{4}, t^{6}+t^{7}\right]\right]$, and verify that the generating function of its Euler characteristics matches the HOMFLY polynomial of the $(2,13)$ cable of the right-handed trefoil knot. 
Remark. It is natural to promote the left hand side of Conjecture 2 to an integral against the weight polynomial; the sum now computes what may be regarded as the homology of a bigraded space. On the other hand, the HOMFLY polynomial is known to arise as the Euler characteristic of the cohomology of a bigraded complex $[\mathrm{KR}]$. We will state a homological version of Conjecture 2 in a subsequent article [ORS].

Acknowledgements. We are grateful to Rahul Pandharipande for suggesting this area of study during a class for which the notes may be found on his website $[\bar{P}]-$ and for advice throughout the project. We have also enjoyed discussions with Margaret Doig, Eduardo Esteves, Paul Hacking, Jesse Kass, Jacob Rasmussen, Giulia Saccà, Sucharit Sarkar, Richard Thomas, and Kevin Wilson, and thank the referees for many helpful comments. A.O. was partially supported by NSF grant DMS-0111298.

\section{SMOOTH POINTS, NODES, AND CUSPS}

We illustrate the conjecture with some elementary examples. Denote by $C_{2, n}$ the formal germ at the origin of the curve cut out by $y^{2}=x^{n}$, and by $\mathcal{O}_{2, n}$ its ring of functions. The link of this singularity is the right-handed $(2, n)$ torus link $T_{2, n}$. The first few of these:

$$
T_{2,0}=\bigcirc \bigcirc \quad T_{2,1}=\bigcirc \quad T_{2,2}=\text { () } T_{2,3}=\gtrsim
$$

Computing $\mathbf{P}\left(T_{2, n}\right)$ is an elementary exercise in the skein relation: smoothing a crossing yields $T_{2, n-1}$ and switching a crossing gives $T_{2, n-2}$. This yields the recurrence

$$
\mathbf{P}\left(T_{2, n}\right)=-a\left(q-q^{-1}\right) \mathbf{P}\left(T_{2, n-1}\right)+a^{2} \mathbf{P}\left(T_{2, n-2}\right)
$$

$T_{2,1}$ is the unknot, and $T_{2,0}$ is two unlinked circles. It is immediate from the skein relation that the HOMFLY polynomial of $n$ unlinked circles is $\left(\left(a-a^{-1}\right) /\left(q-q^{-1}\right)\right)^{n-1}$. Thus:

$$
\begin{aligned}
& \mathbf{P}\left(T_{2,0}\right)=\frac{a-a^{-1}}{q-q^{-1}} \\
& \mathbf{P}\left(T_{2,1}\right)=1 \\
& \mathbf{P}\left(T_{2,2}\right)=-a\left(q-q^{-1}\right)+\frac{a^{3}-a}{q-q^{-1}} \\
& \mathbf{P}\left(T_{2,3}\right)=a^{2} q^{2}+a^{2} q^{-2}-a^{4}
\end{aligned}
$$

We now compute the integral of Conjecture 2 for $n=1,2,3$.

Example 3. As $y^{2}=x$ is smooth at the origin, the Milnor number is $\mu=0$. The ring $\mathcal{O}_{2,1}=\mathbb{C}[[t]]$ has ideals $\left(t^{i}\right)$ for $i \in \mathbb{N}$. Then the conjecture asserts

$$
1=(a / q)^{0}\left(1-q^{2}\right) \sum_{i=0}^{\infty} q^{2 i}
$$

Example 4. At the origin, $y^{2}=x^{2}$ has a node, so the Milnor number is $\mu=1$. We parameterize by $t_{1}=x-y$ and $t_{2}=x+y$ to write $\mathcal{O}_{2,2}=\mathbb{C}[[x, y]] /\left(x^{2}-y^{2}\right)=\mathbb{C}\left[\left[t_{1}, t_{2}\right]\right] /\left(t_{1} t_{2}\right)$. The finite colength ideals of this ring are:

$$
\begin{aligned}
& \left(t_{1}^{k}+\lambda t_{2}^{i-k}\right) \quad \text { for } 1 \leq k<i \text { and } \lambda \in \mathbb{C}^{*} \\
& \left(t_{1}^{k}, t_{2}^{i-k+1}\right) \quad \text { for } 1 \leq k \leq i
\end{aligned}
$$


In each case the variable $i$ gives the colength of the ideal. Each component of the space of ideals of the second type is $\mathbb{C}^{*}$ and thus has Euler characteristic zero. Thus the conjecture asserts

$$
-a\left(q-q^{-1}\right)+\frac{a^{3}-a}{q-q^{-1}}=(a / q)^{1}\left(1-q^{2}\right)\left(1+\left(1-a^{2}\right) \sum_{i=1}^{\infty} i q^{2 i}\right)
$$

Example 5. At the origin, $y^{2}=x^{3}$ has a cusp, so the Milnor number is $\mu=2$. The ideals of the ring $\mathcal{O}_{2,3}=\mathbb{C}\left[\left[t^{2}, t^{3}\right]\right]$ are:

$$
\begin{array}{ll}
(1) & \text { for } i \geq 2 \text { and all } \lambda \in \mathbb{C} \\
\left(t^{i}+\lambda t^{i+1}\right) & \text { for } i \geq 1
\end{array}
$$

Each component of the space of ideals of the second type is $\mathbb{C}$ and thus has Euler characteristic 1. Thus the conjecture asserts

$$
a^{2} q^{2}+a^{2} q^{-2}-a^{4}=(a / q)^{2}\left(1-q^{2}\right)\left(1+\sum_{i=2}^{\infty} q^{2 i}+\left(1-a^{2}\right) \sum_{i=1}^{\infty} q^{2 i}\right)
$$

\section{Specialization to the Alexander polynomial}

In this Section, we show Conjecture 2 holds in the limit $a=-1$ :

Proposition 6. Let $C$ be the germ of a plane curve singularity, and $L_{C}$ its link. Then

$$
\left.\mathbf{P}\left(L_{C}\right)\right|_{a=-1}=\lim _{a \rightarrow-1}(a / q)^{\mu}\left(1-q^{2}\right) \int_{C^{[\star]}} q^{2 l}\left(1-a^{2}\right)^{m-1} d \chi
$$

Both sides of the above equality have simpler expressions. The left hand side is the AlexanderConway polynomial, denoted $\nabla\left(L_{C}\right)$, as can be seen by specializing the skein relations:

$$
\begin{aligned}
\nabla(\nwarrow)-\nabla\left(\lambda^{\Uparrow}\right) & =\left(q-q^{-1}\right) \nabla()() \\
\nabla(\bigcirc) & =1
\end{aligned}
$$

Since the integrand on the right hand side vanishes unless $m=1$, we integrate only over the principal ideals of finite colength. Observe that $\operatorname{dim} \mathcal{O}_{C} / f \mathcal{O}_{C}<\infty$ if and only if $f$ is regular, i.e., neither zero nor a zero divisor; we write $\mathcal{O}_{C}^{\prime}$ for the set of regular elements. Thus finite colength ideals are parameterized by $\mathcal{O}_{C}^{\prime} / \mathcal{O}_{C}^{*}$ and Proposition 6 is equivalent to:

$$
\nabla\left(L_{C}\right)=\left(1-q^{2}\right)(-q)^{-\mu} \int_{\mathcal{O}_{C}^{\prime} / \mathcal{O}_{C}^{*}} q^{2 l} d \chi
$$

where at a point of $\mathcal{O}_{C}^{\prime} / \mathcal{O}_{C}^{*}$ represented by a function $f$, the function $l$ takes the value $\operatorname{dim} \mathcal{O}_{C} / f \mathcal{O}_{C}$.

We will derive Equation (9) from a theorem of Campillo, Delgado, and Gusein-Zade which recovers the Alexander polynomial from the extended semigroup [CDG]. Fix a normalization $n: \mathcal{O}_{C} \hookrightarrow \bigoplus_{i=1}^{b} \mathbb{C}\left[\left[z_{i}\right]\right]$, where $b$ is the number of analytic local branches of $C$. Denoting by $\nu_{i}$ the valuation on $\mathbb{C}\left[\left[z_{i}\right]\right]$ which gives the degree of the lowest order term. We have by restriction a map $\nu=\left(\nu_{1}, \ldots, \nu_{b}\right): \mathcal{O}_{C}^{\prime} \rightarrow \mathbb{N}^{b}$. Its image $\Gamma$ is called the semigroup of the curve singularity 2 It depends on the normalization $n$ only up to reordering of the $z_{i}$. Recording both the degree and the coefficient of the lowest order term gives $\bar{\nu}_{i}: \mathbb{C}\left[\left[z_{i}\right]\right] \backslash 0 \rightarrow \mathbb{N} \times \mathbb{C}^{*}$, and by restriction

\footnotetext{
${ }^{2}$ This notion is classical at least when $b=1$, for a thorough discussion we refer to [ZT].
} 
$\bar{\nu}: \mathcal{O}_{C}^{\prime} \rightarrow\left(\mathbb{N} \times \mathbb{C}^{*}\right)^{b}$. The image $\bar{\Gamma}$ is called the extended semigroup. We write $\mathbb{P} \bar{\Gamma}$ for the quotient by the diagonal $\mathbb{C}^{*}$; note that in the case $b=1$ the composition $\Gamma \hookrightarrow \bar{\Gamma} \rightarrow \mathbb{P} \bar{\Gamma}$ is a bijection. Evidently $\nu_{i}: \mathcal{O}_{C}^{\prime} \rightarrow \mathbb{N}$ descends to a function on $\mathbb{P} \bar{\Gamma}$.

Theorem 7. (Campillo-Delgado-Gusein-Zade [CDG]) Let $C$ be the germ of a plane curve singularity with $b$ branches, and let $L$ be its link. If $b>1$, the multivariate Alexander polynomial is given by

$$
\Delta_{L}\left(t_{1}, \ldots, t_{b}\right)=\int_{\mathbb{P} \bar{\Gamma}} t_{1}^{\nu_{1}} \cdots t_{b}^{\nu_{b}} d \chi
$$

For any $b$, the one-variable Alexander polynomial is given by

$$
\Delta_{L}(t)=(1-t) \int_{\mathbb{P} \bar{\Gamma}} t^{\sum \nu_{i}} d \chi
$$

The Alexander polynomials have been normalized so $\Delta_{L}\left(t_{1}, \ldots, t_{b}\right) \in 1+\left(t_{1}, \ldots, t_{b}\right) \mathbb{Z}\left[t_{1}, \ldots, t_{b}\right]$ and $\Delta_{L}(t) \in 1+t \mathbb{Z}[t]$.

Remark. The statement about the one-variable Alexander polynomial in case $b>1$ is not given explicitly in [CDG] but follows immediately by specializing. Indeed, $(1-t) \Delta_{L}(t, \ldots, t)=\Delta_{L}(t)$, see for instance [Ka, Prop. 7.3.14].

Lemma 8. Let $C$ be the germ of a plane curve singularity; let $L$ be its link and $\mu$ its Milnor number. Normalize its Alexander polynomial by requiring $\Delta_{L}(t) \in 1+t \mathbb{Z}[t]$ and its AlexanderConway polynomial $\nabla_{L}(q)$ by the skein relation given above. Then $\Delta_{L}\left(q^{2}\right)=(-q)^{\mu} \nabla_{L}(q)$.

Proof. It is well known that $\Delta_{L}\left(q^{2}\right)= \pm q^{n} \nabla_{L}(q)$. Since $\nabla_{L}(q)=\nabla_{L}(-1 / q)$, the integer $n$ must be the degree of the Alexander polynomial, which Milnor shows to be $\mu[\mathbf{M}]$. To resolve the sign ambiguity, recall that the link of a plane curve singularity may be realized as the closure of a braid in which only positive crossings ( $)$ appear. Van Buskirk has shown that such "positive links have positive Conway polynomials [ $\mathrm{vB}]$," meaning $\nabla_{L}(q)=\sum n_{i}\left(q-q^{-1}\right)^{i}$ for $n_{i} \in \mathbb{N}$. 3

We rewrite Equation (11) as

$$
\nabla_{L}(q)=\left(1-q^{2}\right)(-q)^{-\mu} \int_{\mathbb{P} \bar{\Gamma}} q^{2 \sum \nu_{i}} d \chi
$$

To prove Proposition 6, it remains to match the integrals in Equations (9) and (12). There are two apparent differences: the exponent on $q$ in the integrand, and the space over which we integrate. As a consequence of the following Lemma, applied with $A=\mathcal{O}_{C}$ and $B$ its normalization, in fact $\sum \nu_{i}(f)=\operatorname{dim} \mathcal{O}_{C} / f \mathcal{O}_{C}$ as functions on $\mathcal{O}_{C}^{\prime}$, hence the integrands agree.

Lemma 9. Let $A \hookrightarrow B$ be rings and let $f \in A$ be a non zero divisor in both $A$ and $B$. If $B / A$, $A / f A$, and $B / f B$ have finite length as $A$-modules, then $A / f A$ and $B / f B$ have equal length.

Proof. Consider the diagram

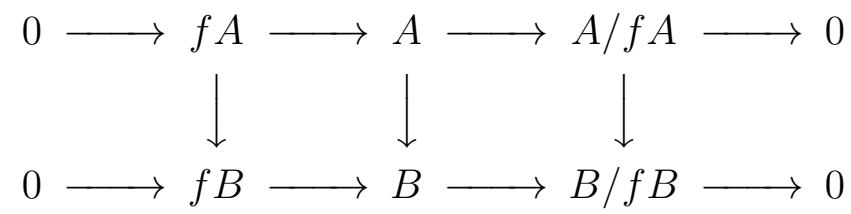

\footnotetext{
${ }^{3}$ In van Buskirk's paper, much more precise conditions on the $n_{i}$ are given.
} 
The Snake Lemma provides the long exact sequence

$$
\begin{aligned}
0 & \rightarrow \operatorname{ker}(f A \rightarrow f B) \rightarrow \operatorname{ker}(A \rightarrow B) \rightarrow \operatorname{ker}(A / f A \rightarrow B / f B) \\
& \rightarrow f B / f A \rightarrow B / A \rightarrow(B / f B) /(A / f A) \rightarrow 0
\end{aligned}
$$

Note that the first two modules in each line are abstractly isomorphic (indeed, the ones on the first line are zero). Since all these modules have finite length, the alternating sum of the lengths is zero, and hence $\operatorname{ker}(A / f A \rightarrow B / f B)$ and $(B / f B) /(A / f A)$ have the same length. But then $A / f A$ and $B / f B$ have the same length.

Remark. The natural map $A / f A \rightarrow B / f B$ is not generally an isomorphism. For example, if $A=\mathbb{C}\left[\left[t^{2}, t^{3}\right]\right] \subset \mathbb{C}[[t]]=B$ and $f=t^{2}$, then $\operatorname{ker}(A / f A \rightarrow B / f B)$ is one dimensional spanned by the class of $t^{3}$, and $\operatorname{coker}(A / f A \rightarrow B / f B)$ is one dimensional spanned by the class of $t$.

Now we compare the spaces $\mathbb{P} \bar{\Gamma}$ and $\mathcal{O}_{C}^{\prime} / \mathcal{O}_{C}^{*}$. Let $\mathcal{O}_{C}^{1 *}$ denote the monic invertible functions, and factor $\bar{\nu}$ as $\mathcal{O}_{C}^{\prime} \stackrel{\pi}{\rightarrow} \mathcal{O}_{C}^{\prime} / \mathcal{O}_{C}^{1 *} \rightarrow \bar{\Gamma}$. We study the fibres. If $\bar{\nu}(f)=\bar{\nu}(g)$ then $\bar{\nu}(f)=\bar{\nu}((1-\lambda) f+\lambda g)$ for any $\lambda \in \mathbb{C}$. If in addition $f$ and $g$ generate the same ideal, then certainly we have an inclusion $((1-\lambda) f+\lambda g) \mathcal{O}_{C} \subset f \mathcal{O}_{C}$. But by Lemma 9 , these ideals have the same colength and thus are equal. We conclude that the fibres of $\bar{\nu}$ and $\pi$ are (infinite dimensional) affine spaces. All maps descend to the quotient by $\mathbb{C}^{*}$ without changing fibres, and if we are willing to take the Euler characteristic of infinite dimensional spaces,

$$
\int_{\mathbb{P} \bar{\Gamma}} q^{2 \sum \nu_{i}} d \chi=\int_{\mathbb{P} \mathcal{O}_{C}^{\prime}} q^{2 \sum \nu_{i}} d \chi=\int_{\mathcal{O}_{C}^{\prime} / \mathcal{O}_{C}^{*}} q^{2 \sum \nu_{i}} d \chi
$$

The Euler numbers of infinite dimensional spaces can made sense of as in [CDG2]. They can also be avoided. At any fixed degree $\sum \nu_{i}=d$, there exists some $N \gg 0$ (twice the $\delta$ invariant will do) such that any principal ideal of colength $d$ can be generated by some $f$ whose expansion as an element of $\bigoplus \mathbb{C}\left[\left[z_{i}\right]\right]$ has no terms of degree larger than $d+N$. We may use the finite dimensional space of such functions in place of $\mathcal{O}_{C}^{\prime}$ to conclude the above equality at degree $d$.

This completes the proof of Proposition 6

\section{THE GENUS EXPANSION}

The HOMFLY polynomial lies in $\mathbb{Z}\left[a^{ \pm 1},\left(q-q^{-1}\right)^{ \pm 1}\right]$; in particular, the skein relation defining it is manifestly invariant under the involution $q \rightarrow-1 / q$. We will show in this Section that the same properties hold for the quantity on the right hand side of Conjecture 2. In the $a \rightarrow 0$ limit of Conjecture 1, this is proven by Pandharipande and Thomas [PT3, Appendix B]. Their approach ultimately rests on Serre duality and the Abel-Jacobi map, and works without modification for the series in Conjecture 2 once note is taken of the following fact from commutative algebra [E]:

Lemma 10. Let $C$ be a reduced integral locally planar curve, and let $\mathcal{F}$ be a torsion free sheaf over $C$. Then for any point $p \in C$ and any line bundle $\mathcal{L}$,

$$
\operatorname{dim}_{\mathbb{C}} \mathcal{F} \otimes \mathbb{C}_{p}=\operatorname{dim}_{\mathbb{C}} \mathcal{H o m}(\mathcal{F}, \mathcal{L}) \otimes \mathbb{C}_{p}
$$

Proof. Taking $A=\mathcal{O}_{C, p}$ and $M=\mathcal{F}_{p}$ it suffices to show $\operatorname{dim} M \otimes_{A} \mathbb{C}=\operatorname{dim} \operatorname{Hom}_{A}(M, A) \otimes_{A} \mathbb{C}$, where $\mathbb{C}$ is the residue field $A / \mathfrak{m}_{p} A$.

Locally $C$ embeds in a surface $S$; let $R=\mathcal{O}_{S, p}$ and $A=R / f R$. As $\mathcal{F}$ was torsion free, any element of $A$ lifts to an element of $R$ which is regular on $M$. Thus $M$ has depth at least 1 over $R$. As $R$ is regular, the Auslander-Buchsbaum theorem guarantees that

$$
\text { proj. } \operatorname{dim}(M)=\operatorname{depth}(R)-\operatorname{depth}(M) \leq 2-1=1
$$


Since $M$ is not a free $R$-module, we have proj. $\operatorname{dim}(M)=1$. Fix a minimal two-term resolution of $M$. Since $M$ is rank zero as an $R$-module, the two terms have the same rank, namely $m:=$ $\operatorname{dim} M \otimes_{R} \mathbb{C}=\operatorname{dim} M \otimes_{A} \mathbb{C}$. On the other hand,

$$
0 \rightarrow R^{m} \rightarrow R^{m} \rightarrow M \rightarrow 0
$$

gives rise to

$$
0=\operatorname{Hom}_{R}(M, R) \rightarrow R^{m} \rightarrow R^{m} \rightarrow \operatorname{Ext}_{R}^{1}(M, R) \rightarrow 0
$$

and thus $\mathbb{C}^{m} \rightarrow \operatorname{Ext}_{R}^{1}(M, R) \otimes \mathbb{C}=\operatorname{Hom}_{A}(M, A) \otimes \mathbb{C}$. We conclude $\operatorname{dim} \operatorname{Hom}_{A}(M, A) \otimes \mathbb{C} \leq$ $\operatorname{dim} M \otimes \mathbb{C}$. As $A$ is Gorenstein, we may dualize again for the reverse inequality.

We pass to a complete curve.

Lemma 11. Let $C$ be a rational curve smooth away from a single point $p$, where it has $b$ analytic local branches. Denote the length functions as $l(I):=\operatorname{dim} \mathcal{O}_{C} / I$ and $l_{p}(I):=\operatorname{dim} \mathcal{O}_{C, p} / I_{p}$, and the function measuring the number of generators by $m(I)=\operatorname{dim} I \otimes \mathbb{C}_{p}$. Then,

$$
\int_{C^{[\star]}} q^{2 l}\left(1-a^{2}\right)^{m-1} d \chi=\left(1-q^{2}\right)^{b-2} \int_{C_{p}^{[\star]}} q^{2 l_{p}}\left(1-a^{2}\right)^{m-1} d \chi
$$

Proof. Consider the map $(C \backslash p)^{[\star]} \times C_{p}^{[\star]} \rightarrow C^{[\star]}$ which takes the (disjoint) union of a scheme supported away from $p$ and a scheme supported at $p$. The map is a bijection with constructible inverse. Denote by $l_{\bar{p}}:(C \backslash p)^{[\star]} \rightarrow \mathbb{N}$ the length function on this space. For $X$ supported at $p$, $Y$ supported away from $p$, and $Z$ their union, we have $l_{p}([X])+l_{\bar{p}}([Y])=l_{C}([Z])$. By definition, $m([Z])=m([X])$. Thus we compute

$$
\int_{C[\star]} q^{2 l_{C}}\left(1-a^{2}\right)^{m-1} d \chi=\int_{C \backslash p^{[\star]}} q^{2 l_{\bar{p}}} d \chi \times \int_{C_{p}^{[\star]}} q^{2 l_{p}}\left(1-a^{2}\right)^{m-1} d \chi
$$

By Equation (1), the first term in the latter product is $\left(1-q^{2}\right)^{-\chi(C \backslash p)}$.

A special case of the BPS calculus of Pandharipande and Thomas is the following:

Proposition 12. (Pandharipande and Thomas [PT3].) Let $C$ be an integral Gorenstein curve of genus $g$, and let $\phi: \overline{\operatorname{Pic}}(C) \rightarrow A$ be a constructible function. Assume that $\phi(F)=\phi(F \otimes L)$ for any line bundle $L$, and moreover that $\phi(F)=\phi\left(\mathcal{H o m}\left(F, \omega_{C}\right)\right)$. Define $n_{h}(C, \phi)$ by the expansion

$$
\int_{C[\star]} q^{2 l} \cdot(\phi \circ A J) d \chi=\sum_{h=-\infty}^{g} q^{2 g-2 h}\left(1-q^{2}\right)^{2 h-2} n_{h}(C, \phi)
$$

Then $n_{h}(C, \phi)=0$ for all $h<0$.

Theorem 13. Let $C_{p}$ be the germ of a plane curve; let $\mu$ be the Milnor number. Then there exist $n_{h}\left(a^{2}\right) \in \mathbb{Z}\left[a^{2}\right]$ such that

$$
(a / q)^{\mu}\left(1-q^{2}\right) \int_{C_{p}[\star]} q^{2 l}\left(1-a^{2}\right)^{m-1} d \chi=a^{\mu} \sum_{h=0}^{\delta} n_{h}\left(a^{2}\right)\left(q^{-1}-q\right)^{2 h+1-b}
$$

The degree in $a^{2}$ of $n_{h}\left(a^{2}\right)$ is at most one less than the multiplicity of the singularity $C_{p}$. 
Proof. As in Lemma 11, let $C$ be a complete rational curve with a unique singularity at $p$ such that $C_{p}$ is the desired germ, and let $m: C^{[n]} \rightarrow \mathbb{N}$ be defined on an ideal sheaf $I$ by $m(I)=\operatorname{dim}_{\mathbb{C}} I \otimes \mathbb{C}_{p}$. Evidently $m(I)$ depends only on the isomorphism class of $I$ as a sheaf, and moreover is unchanged upon tensoring $I$ with a line bundle. By Lemma 10, we also have $m(I)=m\left(\mathcal{H o m}_{C}\left(I, \omega_{C}\right)\right)$. Therefore we may take $\phi=\left(1-a^{2}\right)^{m-1}$ in Proposition 12. To return to the situation for the germ $C_{p}$, we apply Lemma 11 and recall $\mu=2 \delta+1-b[\mathbf{M}]$.

The statement giving the degree of $n_{h}\left(a^{2}\right)$ amounts to the fact that the minimal number of generators of any ideal is bounded by the multiplicity of the singularity. In the unibranch case, this is elementary; in general, see [ $\overline{\mathrm{BH}}$, Exercise 4.6.16].

In the remainder of this Section, we give for completeness an exposition of the proof of Proposition 12. We first recall some properties of torsion free sheaves on Gorenstein curves, which we subsequently use without further comment. We write $F^{*}$ for $\mathcal{H o m}(F, \mathcal{O})$.

Lemma 14. (Hartshorne $[\mathrm{H}]$.) Let $C$ be an integral Gorenstein curve, $F$ a torsion free sheaf. Then higher extensions vanish, $\mathcal{E} x t^{\geq 1}(F, L)=0$, and $F$ is reflexive, $F=\left(F^{*}\right)^{*}$. Serre duality holds in the form $\mathrm{H}^{i}(F)=\mathrm{H}^{1-i}\left(F^{*} \otimes \omega_{C}\right)^{*}$. For $F$ rank one and torsion free, we define its degree $d(F):=\chi(F)-\chi\left(\mathcal{O}_{C}\right)$. This satisfies $d(F)=-d\left(F^{*}\right)$. Moreover if $L$ is any line bundle, $d(F \otimes L)=d(F)+d(L)$.

In great generality, Altman and Kleiman construct a projective scheme $\overline{\mathrm{Pic}}(X)$ whose closed points parameterize rank one, torsion free sheaves on $X[\overline{\mathrm{AK}}]$. We require here only the case where $X=C$ is an integral Gorenstein curve; some statements below are false for general curves.

The space $\overline{\mathrm{Pic}}(C)$ decomposes as a disjoint union $\coprod_{d} \overline{\mathrm{Pic}}_{d}(C)$ indexed by the degree of the sheaves. An Abel-Jacobi map

$$
\begin{aligned}
A J: C^{[d]} & \rightarrow \overline{\operatorname{Pic}}_{d}(C) \\
Z & \mapsto \mathcal{H o m}\left(I_{Z}, \mathcal{O}_{C}\right)
\end{aligned}
$$

can be defined by sending a subscheme to the dual of its ideal sheaf. By Lemma 14, $\mathcal{H o m}\left(\cdot, \mathcal{O}_{C}\right)$ gives a bijection between realizations of a sheaf $I$ as an ideal sheaf, i.e., inclusions $I \hookrightarrow \mathcal{O}_{C}$, and nonzero sections of $I^{*}$. Thus $A J^{-1}(F)=\mathbb{P H}^{0}(F)$.

Fix a line bundle $\mathcal{O}(1)$ of degree 1 on $C$. For $F$ of degree zero we consider

$$
H_{F}(q):=\left(1-q^{2}\right)^{2} \sum q^{2 d} \mathrm{~h}^{0}(F(d))
$$

Since $\phi$ is invariant under tensoring with line bundles,

$$
\int_{C^{[\star]}} q^{2 l}(\phi \circ A J) d \chi=\left(1-q^{2}\right)^{-2} \underset{\overline{\operatorname{Pic}}_{0}(C)}{\int} H \cdot \phi d \chi
$$

Lemma 15. $H_{F}(q)$ is a polynomial of degree at most $4 g$, and $H_{F}(q)=q^{4 g} H_{F^{*} \otimes \omega \otimes \mathcal{O}(2-2 g)}(-1 / q)$.

Proof. The function $h^{0}(F(d))$ is supported in $[0, \infty)$ and is equal to $d+1-g$ in $(2 g-2, \infty)$. Inside $[0,2 g-2]$, it increases by either 0 or 1 at each step. Let

$$
n_{ \pm}(F)=\left\{d \mid 2 \mathrm{~h}^{0}(F(d-1))=\mathrm{h}^{0}(F(d))+\mathrm{h}^{0}(F(d-2)) \pm 1\right\}
$$

Note that $n_{-} \subset[0,2 g]$ and $n_{+} \subset[1,2 g-1]$. The expansion

$$
H_{F}(q)=\sum_{d \in n_{-}(F)} q^{2 d}-\sum_{d \in n_{+}(F)} q^{2 d}
$$


establishes the polynomiality and degree of $H$. Moreover, by Serre duality and the Riemann-Roch formula, we have $d \in n_{ \pm}\left(F^{*} \otimes \omega \otimes \mathcal{O}(2-2 g)\right) \Longleftrightarrow 2 g-d \in n_{ \pm}(F)$. This establishes the desired symmetry.

Since $\phi$ is invariant under the involution $F \mapsto F^{*} \otimes \omega \otimes \mathcal{O}(2-2 g)$ of $\overline{\text { Pic }}_{0}$, we can integrate the previous Lemma to find that $Z_{\phi}(q):=\int{\overline{\operatorname{Pic}_{0}(C)}}_{1} H \cdot \phi d \chi$ is a polynomial of degree at most $4 g$ in $q$ and $Z_{\phi}(q)=q^{4 g} Z_{\phi}(-1 / q)$. To finish the proof of Proposition 12, it remains only to observe that $\left\{q^{2 g-2 h}\left(1-q^{2}\right)^{2 h}\right\}$ span the space of such functions.

\section{THE CURVE $y^{k}=x^{n}$}

We consider now the singularity at the origin of $y^{k}=x^{n}$ for $k, n$ relatively prime. The complete local ring is $\mathbb{C}\left[\left[t^{k}, t^{n}\right]\right]$, and the corresponding knot is the $(k, n)$ torus knot. Jones has computed its HOMFLY polynomial.

Theorem 16 (Jones).

$$
\mathbf{P}\left(T_{k, n}\right)=\frac{\left(1-q^{2}\right)(a / q)^{(k-1)(n-1)}}{\left(1-q^{2 k}\right)\left(1-a^{2}\right)} \sum_{j=0}^{k-1}(-1)^{j} \frac{\left(q^{2}\right)^{j n+(k-1-j)(k-j) / 2}}{[j] ![k-1-j] !} \prod_{i=j+1-k}^{j}\left(q^{2 i}-a^{2}\right)
$$

where $[0] !=1$ and $[r] !=\left(1-q^{2 r}\right)[r-1]$ !

The Milnor number of this singularity is $\mu=(k-1)(n-1)$. After rearranging the normalization factors, Conjecture 2] asserts:

$$
\int_{C_{p}^{[\star]}} q^{2 l}\left(1-a^{2}\right)^{m} d \chi=\frac{1}{1-q^{2 k}} \sum_{j=0}^{k-1}(-1)^{j} \frac{\left(q^{2}\right)^{j n+(k-1-j)(k-j) / 2}}{[j] ![k-1-j] !} \prod_{i=j+1-k}^{j}\left(q^{2 i}-a^{2}\right)
$$

To compute the left hand side we use a torus action. $\mathbb{C}^{*}$ acts on $\mathbb{C}\left[\left[t^{k}, t^{n}\right]\right]$ by scaling $t$. The action lifts to the Hilbert scheme, and preserves the functions $l, m$ measuring length and number of generators. The integral with respect to Euler characteristic of an $\mathbb{C}^{*}$-equivariant function may always be computed on the fixed locus of the $\mathbb{C}^{*}$ action since the remainder of the space will be fibred by $\mathbb{C}^{*}$ and hence contribute zero to the Euler characteristic. Diagonalizing the $\mathbb{C}^{*}$ action on a fixed ideal will yield monomial generators; conversely all the monomial ideals are fixed. There are countably many of these, and only finitely many with colength below any given bound. Therefore:

$$
\int_{C_{p}^{[\star]}} q^{2 l}\left(1-a^{2}\right)^{m} d \chi=\sum_{J \text { monomial }} q^{2 \operatorname{dim}_{\mathbb{C}} \mathcal{O} / J}\left(1-a^{2}\right)^{m(J)}
$$

Lemma 17. The monomial ideals are enumerated by the following function.

$$
\sum_{J \text { monomial }} q^{2 \operatorname{dim}_{\mathbb{C}} \mathcal{O} / J}\left(1-a^{2}\right)^{m(J)}=\frac{1}{1-q^{2 k}} \operatorname{res}_{\xi=0} \frac{1}{\xi^{n+1}} \prod_{i=0}^{k-1}\left(1+\left(1-a^{2}\right) \frac{\xi q^{2 i}}{1-\xi q^{2 i}}\right)
$$

Proof. Monomial ideals of $\mathbb{C}[[x, y]]$ can be matched with staircases $[\mathrm{Br}, \mathrm{I}]$. We proceed similarly. Consider the map

$$
\begin{aligned}
\mathbb{N} \times\{0, \ldots, k-1\} & \rightarrow \text { monomials } \in \mathcal{O} \\
(\alpha, \beta) & \mapsto t^{\alpha k+\beta n}
\end{aligned}
$$


It follows from the Chinese remainder theorem that this is a bijection. Monomial ideals are in 1-1 correspondence with sequences $\phi=\phi_{k-1} \leq \phi_{k-2} \leq \ldots \leq \phi_{0} \leq \phi_{k-1}+n$ via the correspondence

$$
\phi \leftrightarrow\left\{(\alpha, \beta) \mid \alpha>\phi_{\beta}\right\}
$$

The number of generators of the ideal is the number of inequalities above which are strict. The cardinality of the complement of the ideal is "the number of boxes under the staircase," or $\sum \phi_{i}$.

Regarding the $\phi_{i}$ as the lengths of the rows of the staircase, the formula in the lemma enumerates the staircases column by column. The leading term $\left(1-q^{2 k}\right)^{-1}$ accounts for the leading columns of full height $k$. The term $i$ in the product corresponds to columns of height $i$. The number of different column heights is equal to the number of inequalities in $\phi$. The residue enforces the condition that there should be exactly $n$ columns of height less than $k$.

Example 18. We give the staircase of $\left(t^{21}, t^{23}, t^{24}\right) \subset \mathbb{C}\left[\left[t^{4}, t^{5}\right]\right]$. Bold numbers correspond to monomials in the ideal.

\begin{tabular}{|c|c|c|c|c|c|c|c}
\hline 15 & 19 & $\mathbf{2 3}$ & $\mathbf{2 7}$ & $\mathbf{3 1}$ & $\mathbf{3 5}$ & $\mathbf{3 9}$ & $\mathbf{4 3}$ \\
\hline 10 & 14 & 18 & 22 & $\mathbf{2 6}$ & $\mathbf{3 0}$ & $\mathbf{3 4}$ & $\mathbf{3 8}$ \\
\hline 5 & 9 & 13 & 17 & $\mathbf{2 1}$ & $\mathbf{2 5}$ & $\mathbf{2 9}$ & $\mathbf{3 3}$ \\
\hline 0 & 4 & 8 & 12 & 16 & 20 & $\mathbf{2 4}$ & $\mathbf{2 8}$ \\
\hline
\end{tabular}

Example 19. The following staircase does not correspond to any ideal of $\mathbb{C}\left[\left[t^{4}, t^{5}\right]\right]$, because $28=$ $23+5$. This occurs because the staircase does not descend quickly enough.

\begin{tabular}{|c|c|c|c|c|c|c|c|c}
\hline 15 & 19 & $\mathbf{2 3}$ & $\mathbf{2 7}$ & $\mathbf{3 1}$ & $\mathbf{3 5}$ & $\mathbf{3 9}$ & $\mathbf{4 3}$ & $\mathbf{4 7}$ \\
\hline 10 & 14 & 18 & 22 & $\mathbf{2 6}$ & $\mathbf{3 0}$ & $\mathbf{3 4}$ & $\mathbf{3 8}$ & $\mathbf{4 2}$ \\
\hline 5 & 9 & 13 & 17 & $\mathbf{2 1}$ & $\mathbf{2 5}$ & $\mathbf{2 9}$ & $\mathbf{3 3}$ & $\mathbf{3 7}$ \\
\hline 0 & 4 & 8 & 12 & 16 & 20 & 24 & 28 & $\mathbf{3 2}$ \\
\hline
\end{tabular}

We evaluate the residue in Lemma 17 by summing over the other singularities of the expression. These occur precisely at $\xi=q^{-2 j}$ for $j=0,1, \ldots, k-1$. Note that

$$
\operatorname{res}_{z=1 / w} \frac{1}{1-w z}=-\frac{1}{w}
$$

in order to evaluate the residue:

$$
\frac{1}{1-q^{2 k}} \sum_{j=0}^{k-1} q^{2(n+1) j}\left(\prod_{i=0}^{j-1} \frac{1}{1-q^{2(i-j)}}\right) q^{-2 j}\left(\prod_{i=j+1}^{k-1} \frac{1}{1-q^{2(i-j)}}\right)\left(\prod_{i=0}^{k-1} 1-a^{2} q^{2(i-j)}\right)
$$

It remains to collect signs and powers of $q$ in order to prove:

Theorem 20. Let $\operatorname{gcd}(k, n)=1$. Let $C$ be the curve cut out by $y^{k}=x^{n}$ and let $p$ be the origin; $\mu=(k-1)(n-1)$ is the Milnor number of this singularity, its link is the $k, n$ torus $k n o t$, and

$$
\mathbf{P}(k, n \text { torus knot })=(a / q)^{\mu}\left(1-q^{2}\right) \int_{C_{p}[\star]} q^{2 l}\left(1-a^{2}\right)^{m-1} d \chi
$$

Corollary 21. Let $C$ be a rational curve, smooth away from a point $p$, and formally isomorphic at p to Spec $\mathbb{C}[[x, y]] /\left(y^{k}=x^{n}\right)$. Write $\left(\begin{array}{l}b \\ c\end{array}\right)_{q^{2}}$ for $\frac{[b] !}{[c] ![b-c] !}$. Then

$$
\left(1-q^{2}\right)^{2} \int_{C[\star]} q^{2 l} d \chi=\frac{\left(\begin{array}{c}
k+n \\
k
\end{array}\right) q^{2}}{\left(\begin{array}{c}
k+n \\
1
\end{array}\right) q^{2}}
$$


Proof. We have proven Conjecture 2 in the case of the singularity in question, which implies Conjecture 1. Substituting in, we see

$$
\begin{aligned}
\left(1-q^{2}\right)^{2} \int_{C^{[\star]}} q^{2 l} d \chi & =\frac{\left(1-q^{2}\right)}{\left(1-q^{2 k}\right)} \sum_{j=0}^{k-1}(-1)^{j} \frac{\left(q^{2}\right)^{j n+(k-1-j)(k-j) / 2}}{[j] ![k-1-j] !} \prod_{i=j+1-k}^{j} q^{2 i} \\
& =\frac{\left(1-q^{2}\right)}{[k] !} \sum_{j=0}^{k-1}\left(\begin{array}{c}
k-1 \\
j
\end{array}\right)_{q^{2}} q^{j(j-1)}\left(-q^{2(n+1)}\right)^{j}
\end{aligned}
$$

Now we use the "Newton formula for Gaussian binomials"

$$
\sum_{r=0}^{s} q^{r(r-1)}\left(\begin{array}{l}
s \\
r
\end{array}\right)_{q^{2}} t^{r}=\prod_{r=0}^{s-1}\left(1+q^{2 r} t\right)
$$

to deduce

$$
\left(1-q^{2}\right)^{2} \int_{C^{[\star]}} q^{2 l} d \chi=\frac{\left(1-q^{2}\right)}{[k] !} \prod_{j=0}^{k-2}\left(1-\left(q^{2}\right)^{n+j+1}\right)=\frac{\left(\begin{array}{c}
k+n \\
k
\end{array}\right)_{q^{2}}}{\left(\begin{array}{c}
k+n \\
1
\end{array}\right)_{q^{2}}}
$$

Setting $q=1$ recovers Beauville's formula $[\bar{B}]$ for the Euler number of the compactified Jacobian.

\section{HILBERT SCHEMES OF UNIBRANCH SINGULARITIES}

Let $C$ be the germ of a unibranch singularity, and fix a normalization $\mathcal{O}=\mathcal{O}_{C} \hookrightarrow \mathbb{C}[[t]]$. Let $\nu: \mathbb{C}[[t]] \rightarrow \mathbb{N}$ be the valuation taking a series to the degree of its lowest degree term. Lemma 9 shows that $\nu(f)=\operatorname{dim}_{\mathbb{C}} \mathcal{O} / f \mathcal{O}$, so $\left.\nu\right|_{\mathcal{O}}$ is independent of the choice of normalization. Let $\Gamma=\nu(\mathcal{O})$ - when appropriate we implicitly exclude 0 from the domain of $\nu$ - then $\Gamma$ is a cofinite 4 subset of $\mathbb{N}$ closed under addition. We employ the filtration $F_{k} \mathcal{O}=\{f \in \mathcal{O} \mid \nu(f) \geq k\}$.

Lemma 22. $\operatorname{dim} F_{k} \mathcal{O} / F_{k+1} \mathcal{O} \leq 1$.

If $J$ an ideal of $\mathcal{O}$, then $\nu(J) \subset \mathbb{N}$ is a semigroup ideal: $\nu(J)+\Gamma \subset \nu(J)$.

Corollary 23. Let $J$ be an ideal of $\mathcal{O}$. Then $\operatorname{dim}_{\mathbb{C}} \mathcal{O} / J=\# \nu(\mathcal{O}) \backslash \nu(J)$.

Proof. As $\operatorname{dim}_{\mathbb{C}} \mathcal{O} / J$ is finite, $\operatorname{dim} \mathcal{O} / J=\sum \operatorname{dim} F_{n} \mathcal{O} /\left(F_{n} \mathcal{O} \cap J+F_{n+1} \mathcal{O}\right)$. This contributes 1 exactly when there is a ring element with valuation $n$, but no ideal element with valuation $n$; i.e., for $n \in \nu(\mathcal{O}) \backslash \nu(J)$.

For elements $a_{0}, \ldots, a_{k} \in \Gamma$, we denote the semigroup ideal they generate by

$$
\left(a_{0}, \ldots, a_{k}\right)_{\Gamma}=\left\{a_{i}+\gamma_{i} \mid \gamma_{i} \in \Gamma\right\}
$$

Corollary 24. Let $J$ be an ideal of $\mathcal{O}$. For $f_{0}, \ldots, f_{k} \in J$,

$$
\left(\nu\left(f_{0}\right), \ldots, \nu\left(f_{k}\right)\right)_{\Gamma}=\nu(J) \Longrightarrow\left(f_{0}, \ldots, f_{k}\right)=J
$$

Proof. Let $J^{\prime}$ be the ideal generated by the $f_{i}$. Since $J^{\prime} \subset J$, surely $\nu\left(J^{\prime}\right) \subset \nu(J)$. But

$$
\nu\left(J^{\prime}\right) \supset\left(\nu\left(f_{0}\right), \ldots, \nu\left(f_{k}\right)\right)_{\Gamma}=\nu(J)
$$

Thus $\operatorname{dim} J / J^{\prime}=\operatorname{dim} \mathcal{O} / J^{\prime}-\operatorname{dim} \mathcal{O} / J=\# \Gamma \backslash \nu\left(J^{\prime}\right)-\# \Gamma \backslash \nu(J)=0$.

\footnotetext{
${ }^{4}$ If not, then the fraction field of $\mathcal{O}$ would be some $k\left(\left(t^{r}\right)\right) \subsetneq k((t))$, but $\mathcal{O}$ and its normalization $k[[t]]$ must share the same fraction field. For the theory of unibranch singularities, we refer to the book of Zariski and Teissier [ZT].
} 
Remark. The converse is false. The ring $\mathcal{O}=k\left[\left[t^{4}, t^{6}+t^{7}\right]\right]$ has semigroup $\langle 4,6,13\rangle$. Its maximal ideal $M=\left(t^{4}, t^{6}+t^{7}\right)$ has semigroup ideal $\nu(M)=(4,6,13)_{\Gamma}$. In fact, there is no ideal $J$ with $\nu(J)=(4,6)_{\Gamma}$ : any such ideal contains $t^{4}, t^{6}+t^{7}$, hence $\left(t^{6}+t^{7}\right)^{2}-\left(t^{4}\right)^{3}=2 t^{13}+t^{14}$.

We write $\Gamma^{[\star]}$ for the set of semigroup ideals of $\Gamma$, and view $\nu$ as a constructible map

$$
\nu: C^{[\star]} \rightarrow \Gamma^{[\star]}
$$

which lets us define a stratification $C^{[j]}=\nu^{-1}(\mathfrak{j})$. Corollary 23 shows that this is a sub-stratification of the usual one by length: $C^{[\mathrm{j}]} \subset C^{[\# \Gamma \backslash \mathrm{j}]}$.

Remark. Teissier $[\overline{\mathrm{ZT}}]$ constructs a $\mathbb{C}^{*}$-equivariant deformation $\mathcal{C} \rightarrow \mathbb{A}^{1}$ whose generic fibre is $C$ and whose special fibre is the not-necessarily-planar $C_{\Gamma}=\operatorname{Spec} \mathbb{C}[[\Gamma]]$. The central fibre carries a natural $\mathbb{C}^{*}$ action. Lifting the action to the Hilbert scheme, $\left(C_{\Gamma}{ }^{\left[{ }^{*}\right]}\right)^{\mathbb{C}^{*}}=\Gamma^{[\star]}$. The map $\nu$ amounts to taking the $t \rightarrow 0$ limit of the $\mathbb{C}^{*}$ action on the relative Hilbert scheme of $\mathcal{C} / \mathbb{A}^{1}$.

Lemma 25. For $g \in \Gamma$ there exists a unique element $\tau_{g} \in \mathcal{O} \subset \mathbb{C}[[t]]$ of the form

$$
\tau_{g}=t^{g}+\sum_{g<i \notin \Gamma} c_{i} t^{i}
$$

Proof. Since $g \in \Gamma$, there is some element $\tilde{\tau} \in \mathcal{O}$ with leading term $t^{g}$. Terms in $\tilde{\tau}$ of degrees in $\Gamma$ may be successively removed; the process converges since $\mathcal{O}$ is complete. Given $\tau_{g} \neq \tau_{g}^{\prime}$ of the form prescribed, we have $\nu\left(\tau_{g}-\tau_{g}^{\prime}\right) \neq \Gamma$, which is absurd.

Definition 26. Fix $a_{0}, \ldots, a_{k} \in \Gamma$. Use $\Sigma_{\lambda}:=\Gamma_{>a_{\lambda}} \backslash\left(a_{0}, \ldots, a_{k}\right)_{\Gamma}$ to index the set of indeterminates $\mathbf{S}:=\left\{S_{\lambda, i} \mid i \in \Sigma_{\lambda}\right\}$. Let $V_{a_{0}, \ldots, a_{k}}:=\operatorname{Spec} \mathbb{C}[\mathbf{S}]$, and define $\mathbf{f}_{\lambda} \in \mathcal{O}[\mathbf{S}]$ by

$$
\mathbf{f}_{\lambda}:=\tau_{a_{\lambda}}+\sum_{i \in \Sigma_{\lambda}} \tau_{i} S_{\lambda, i}
$$

and form the ideal $\mathcal{J}:=\left(\mathbf{f}_{0}, \ldots, \mathbf{f}_{k}\right) \subset \mathcal{O}[\mathbf{S}]$. Consider the subvariety

$$
U_{a_{0}, \ldots, a_{k}}:=\left\{s \in V_{a_{0}, \ldots, a_{k}} \mid \nu\left(\left.\mathcal{J}\right|_{s}\right)=\left(a_{0}, \ldots, a_{k}\right)_{\Gamma}\right\}
$$

As we always have $\left(a_{0}, \ldots, a_{k}\right)_{\Gamma} \subset \nu\left(\left.\mathcal{J}\right|_{s}\right), U_{a_{0}, \ldots, a_{k}}$ is equivalently described as the locus where $\operatorname{dim} \mathcal{O} / \mathcal{J}_{s}=\# \Gamma \backslash\left(a_{0}, \ldots, a_{k}\right)_{\Gamma}$. As the Hilbert polynomial is constant and the base is reduced, $\mathcal{O}[\mathbf{S}] / \mathcal{J}$ is flat over $U_{a_{0}, \ldots, a_{k}}$. Thus we get a map

$$
\Psi_{a_{0}, \ldots, a_{k}}: U_{a_{0}, \ldots, a_{k}} \rightarrow C^{\left[\left(a_{0}, \ldots, a_{k}\right)_{\Gamma}\right]} \subset C^{[\star]}
$$

Theorem 27. $\Psi_{a_{0}, \ldots, a_{k}}: U_{a_{0}, \ldots, a_{k}} \rightarrow C^{\left[\left(a_{0}, \ldots, a_{k}\right)_{\Gamma}\right]}$ is bijective.

Proof. Let $\mathfrak{j}=\left(a_{0}, \ldots, a_{k}\right)_{\Gamma}$. Consider a closed point $\left(s_{\lambda, i}\right)$ in the preimage of $C^{[j]}$. This corresponds to an ideal $J=\left(f_{0}, \ldots, f_{k}\right)$ where $f_{i}=\tau_{a_{\lambda}}+\sum_{i \in \Sigma_{\lambda}} \tau_{i} s_{\lambda, i}$. Suppose $J$ is also the ideal corresponding to $\left(s_{\lambda, i \in \Sigma_{\lambda}}^{\prime}\right)$, hence has generators $f_{\lambda}^{\prime}=\tau_{a_{\lambda}}+\sum_{i \in \Sigma_{\lambda}} \tau_{i} s_{\lambda, i}^{\prime}$. Now $f_{\lambda}-f_{\lambda}^{\prime} \in J$, but on the other hand $\nu\left(f_{\lambda}-f_{\lambda}^{\prime}\right) \in \Gamma \backslash \mathfrak{j}$ unless $f_{\lambda}=f_{\lambda}^{\prime}$. Thus the map is injective.

For surjectivity, fix an ideal $J$ with $\nu(J)=\mathfrak{j}$. Choose lifts of $f_{\lambda} \in J$ of the $a_{\lambda}$. A generating set will still generate if we modify $f_{\lambda} \rightarrow u f_{\lambda}+\sum_{\nu \neq \lambda} v f_{\nu}$ for invertible $u$ and arbitrary $v$. Iteratively removing terms of the form $t^{n}$ for $n \in \mathfrak{j} \backslash \lambda$ from $f_{\lambda}$ converges to yield generators of $J$ of the form required by Definition 26 .

Remark. As defined, the $V_{a_{0}, \ldots, a_{k}}$ depend on the choice of generators of the semigroup ideal $\left(a_{0}, \ldots, a_{k}\right)_{\Gamma}$. However, a semigroup ideal has a unique minimal generating set; henceforth if we write $V_{\mathrm{j}}$ to mean that the minimal generating set of the semigroup ideal is chosen. On the other 
hand, while it may likewise seem that $U_{a_{0}, \ldots, a_{k}}$ depends on the choice of generators, Theorem 27 implies that all choices yield spaces which biject onto $C^{[j]}$.

Caution. The function giving the number of generators need not be constant on the $U_{j}$.

For computations, the following consequence of Lemma 9 is useful.

Corollary 28. Let $\Gamma \subset \mathbb{N}$ be a semigroup with $\# \mathbb{N} \backslash \Gamma<\infty$. For $i \in \Gamma$, $\# \Gamma \backslash(i+\Gamma)=i$. More generally, let $\Gamma \subset \Delta \subset \mathbb{N}$, and suppose $i+\Delta \subset \Gamma$. Then

$$
\# \Gamma \backslash(i+\Delta)=i-\# \Delta \backslash \Gamma
$$

Proof. Consider $\mathbb{C}[\Gamma]$, the ring with generators $\left\{x^{\gamma}\right\}_{\gamma \in \Gamma}$ and relations $x^{\gamma_{1}} x^{\gamma_{2}}=x^{\gamma_{1}+\gamma_{2}}$. Now apply Lemma 9 to $\mathbb{C}[\Gamma] \hookrightarrow \mathbb{C}[x]$ and $x^{i} \in \mathbb{C}[\Gamma]$ to see $\# \Gamma \backslash(i+\Gamma)=i$. The final statement follows from $\# \Gamma \backslash(i+\Delta)+\#(i+\Delta) \backslash(i+\Gamma)=\# \Gamma \backslash(i+\Gamma)=i$ and $\#(i+\Delta) \backslash(i+\Gamma)=\# \Delta \backslash \Gamma$.

\section{The Singularity WiTH SEMigRouP $\langle 4,6,13\rangle$}

We consider now the ring $\mathcal{O}=\mathbb{C}\left[\left[t^{4}, t^{6}+t^{7}\right]\right]$ and the singularity $C=\operatorname{Spec} \mathcal{O}$. Let us calculate the semigroup. As $\left(t^{6}+t^{7}\right)^{2}-\left(t^{4}\right)^{3}=t^{13}(2+t)$ we see 4,6,13 $\in \nu(\mathcal{O})$. Suppose there is $P(x, y) \in \mathbb{C}[[x, y]]$ such that $P(t)=P\left(t^{4}, t^{6}+t^{7}\right)$ has leading term $t^{15}$. Then certainly $P(x, y)$ must have two monomials, $x^{a} y^{b}$ and $x^{c} y^{d}$, such that $4 a+6 b=4 c+6 d<15$; moreover their leading terms must cancel when evaluated at $x=t^{4}, y=t^{6}+t^{7}$. By inspection, the first condition is only satisfied for $4 a+6 b=12$, but in this case we have already seen that the leading term of $P(t)$ is $t^{13}$. So $15 \notin \nu(\mathcal{O})$, and the semigroup is

$$
\Gamma=\langle 4,6,13\rangle=\{0,4,6,8,10,12,13,14,16,17, \ldots\}
$$

In fact, Zariski has shown that this is the only plane singularity with this semigroup [ZT].

The link of this singularity is the $(2,13)$ cable of the $(2,3)$ torus knot [EN]. Its HOMFLY polynomial, as calculated by computer, is

$$
\begin{array}{lll}
- & a^{22} & \left(3+4 z^{2}+z^{4}\right) \\
+ & a^{20} & \left(20+70 z^{2}+84 z^{4}+45 z^{6}+11 z^{8}+z^{10}\right) \\
- & a^{18} & \left(39+220 z^{2}+468 z^{4}+496 z^{6}+286 z^{8}+91 z^{10}+15 z^{12}+z^{14}\right) \\
+ & a^{16} & \left(23+179 z^{2}+540 z^{4}+836 z^{6}+726 z^{8}+365 z^{10}+105 z^{12}+16 z^{14}+z^{16}\right)
\end{array}
$$

where $z=q-q^{-1}$. According to Conjecture1, the coefficient of $z^{2 h}$ in the bottom row above is the number $n_{h}$ of Pandharipande and Thomas [PT3, Appendix 2]. In particular, the Euler characteristic of the Jacobian factor of this singularity should be $n_{0}=23$. This was previously calculated by

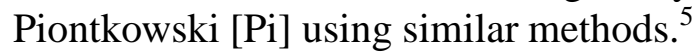

We turn now to the calculation of the integral in Conjecture 2 by using the stratification of Section 6. Moreover we group the semigroup ideals which are isomorphic as $\Gamma$-modules, i.e., differ merely by a shift. Let $\operatorname{Mod}(\Gamma)$ denote the set of $\Gamma$ submodules of $\mathbb{N}$ containing zero. Using

\footnotetext{
5 Indeed, Piontkowski also determines the Euler characteristic of the Jacobian factor for all singularities with semigroups $\langle 4,2 q, s\rangle,\langle 6,8, s\rangle$, and $\langle 6,10, s\rangle$. He does so by constructing a stratification of the Jacobian factor by affine spaces, and suggests that it is unlikely that any other singularities will admit such a stratification. We remark that his list exhausts the algebraic $(2, n)$ cables of $(2, p),(3,4)$ and $(3,5)$ torus knots, or in other words, the $(2, n)$ cables of knots arising from simple singularities.
} 
Theorem 27 and Corollary 28 ,

$$
\int_{C^{[\star]}} q^{2 l}\left(1-a^{2}\right)^{m-1} d \chi=\sum_{\Delta \in \operatorname{Mod}(\Gamma)} q^{-(8-\# \mathbb{N} \backslash \Delta)} \sum_{i+\Delta \subset \Gamma} q^{2 i} \int_{U_{i+\Delta}}\left(1-a^{2}\right)^{m-1} d \chi
$$

The combinatorial data required to compute the right hand side is tabulated in Figure 1, which appears at the end of the article. We compute the rightmost integral by determining the spaces $U_{i+\Delta}$, together with their stratifications by the number of generators.

Lemma 29. Let $\Gamma=\langle 4,6,13\rangle$, and consider $\Delta \in \operatorname{Mod}(\Gamma)$. Let $0=\alpha_{0}<\alpha_{1}<\ldots$ be its minimal set of generators. Choose arbitrary $f_{0}, f_{1}, \ldots \in k[[t]]$ with degrees $\alpha_{0}, \alpha_{1}, \ldots$ Then if $\eta=\nu\left(\sum f_{i} \phi_{i}\right) \notin \Delta$ for $\phi_{i} \in \mathcal{O}$, then:

- $\alpha_{1}=2$ and $1,3 \notin \Delta$

- $\eta \in\{7,9,11,15\}$

- $\nu\left(f_{0} \phi_{0}\right)=\nu\left(f_{1} \phi_{1}\right)=\min \left\{\nu\left(f_{i} \phi_{i}\right)\right\}$

Proof. Assume there exist $\phi_{i} \in \mathcal{O}$ such that $\eta=\nu\left(\sum f_{i} \phi_{i}\right) \notin \Delta$. In particular, we must have $\nu\left(\sum f_{i} \phi_{i}\right)>\min \nu\left(f_{i} \phi_{i}\right)$, thus the lowest degree terms must cancel, thus there must be at least two of them. Say they are $f_{j}$ and $f_{k}$; let $\alpha_{j}<\alpha_{k}$. We have

$$
\eta>\alpha_{k}+\nu\left(\phi_{k}\right)=\alpha_{j}+\nu\left(\phi_{j}\right)
$$

We cannot have $\nu\left(\phi_{k}\right)=0$ since $\alpha_{k}$ is a necessary generator; thus $\nu\left(\phi_{k}\right) \geq 4$. Since $\nu\left(\phi_{j}\right)>$ $\nu\left(\phi_{k}\right)$ we also have $\nu\left(\phi_{j}\right) \geq 6$. As $\eta>\alpha_{j}+\nu\left(\phi_{j}\right) \geq 6$ and $\eta \notin \Delta$, we must have $\eta \in\{7,9,11,15\}$. Since $\eta \notin \Delta$, no odd number less than $\eta-2$ can be in $\Delta$. This implies that $1,3 \notin \Delta$ and $a_{j}, a_{k}$ are even. This can only happen if $a_{j}=0$ and $a_{k}=2$.

Lemma 30. Given two series

$$
\begin{aligned}
& f_{0}=1+a_{1} t+a_{3} t^{3}+\ldots \\
& f_{1}=t^{2}\left(1+b_{1} t+b_{3} t^{3}+\ldots\right)
\end{aligned}
$$

we see that

$$
\begin{array}{lll}
\operatorname{deg}_{t}\left(\left(t^{6}+t^{7}\right) f_{0}-t^{4} f_{1}\right) \geq 7 & \text { with equality unless } & b_{1}-a_{1}=1 \\
\operatorname{deg}_{t}\left(\left(t^{6}+t^{7}\right) f_{1}-t^{8} f_{0}\right) \geq 9 & \text { with equality unless } & a_{1}-b_{1}=1
\end{array}
$$

The equations cannot hold simultaneously, so at least one of the series has the specified degree.

Corollary 31. Let $0 \in \Delta \subset \mathbb{N}$ be a $\Gamma$-module with minimal generators $\alpha_{0}, \alpha_{1}, \ldots$ Choose lifts $f_{0}, f_{1}, \ldots \in \mathbb{C}[[t]]$. Let $\Delta^{\prime}=\nu\left(\left(f_{0}, f_{1}, \ldots\right)\right)$. Then if $\Delta \neq \Delta^{\prime}$, then $\Delta \rightarrow \Delta^{\prime}$ appears on the following list.

- $(0,2) \rightarrow(0,2,7),(0,2,9),(0,2,7,9)$

- $(0,2,5) \rightarrow(0,2,5,7)$

- $(0,2,7) \rightarrow(0,2,7,9)$

- $(0,2,9) \rightarrow(0,2,7,9),(0,2,9,11)$

- $(0,2,11) \rightarrow(0,2,7),(0,2,7,9),(0,2,9,11)$ 
For all modules $\Phi$ not occuring on the list,

$$
\int_{U_{i+\Phi}}\left(1-a^{2}\right)^{m-1} d \chi=\left(1-a^{2}\right)^{m(\Phi)-1}
$$

where $m(\Phi)$ is the number of generators of $\Phi$ as a $\Gamma$-module. Moreover, if $\Phi=(0,2)$ or $(0,2,11)$, then $U_{i+\Phi}=\emptyset$, so

$$
\int_{U_{i+\Phi}}\left(1-a^{2}\right)^{m-1} d \chi=0
$$

Proof. All $\Gamma$ modules are listed in Figure 1. Checking the criterion of Lemma 29 on each of them yields the list of possible $\Delta$. The list of possible $\Delta^{\prime}$ comes from the criterion of Lemma 30, if 0 and 2 are in $\Delta$, then 7 or 9 is in $\Delta^{\prime}$. If a semigroup module $M$ never occurs as one of the $\Delta$ above, Theorem 27 implies that $U_{i+M}$ is an affine space. If $M$ never occurs as one of the $\Delta^{\prime}$, then Theorem 27implies that $\nu(I)=i+M \Longrightarrow m(I)=m(M)$. The final statement of the corollary is immediate from Lemma 30 .

We proceed to analyse the remaining modules. Suppose $\Delta \in \operatorname{Mod}(\Gamma), 0,2 \in \Delta$, and $1,3 \notin \Delta$. Fix $i$ such that $i+\Delta \subset \Gamma$, and let $V_{i+\Delta}$ be the affine space of Definition 26. $V_{i+\Delta}$ has coordinate functions $\mathbf{a}, \mathbf{b}$ giving respectively the coefficient of $x^{i+1}$ of the generator of degree $i$, and the coefficient of $x^{i+3}$ in the generator of degree $i+2$. Let $I$ be an ideal corresponding to some point in $V_{i+\Delta}$ such that $a=\mathbf{a}(I)$ and $b=\mathbf{b}(I)$. Lemma 30 implies that $i+7 \in \nu(I)$ unless $b-a=1$, and $i+9 \in \nu(I)$ unless $a-b=1$. In fact, $b-a$ is identically 1 if and only if $i+7 \notin \Gamma$, identically -1 if and only if $i+9 \notin \Gamma$, and otherwise may assume any value. This may be seen from the explicit form of a general element of $\mathcal{O}$ :

$$
c_{0}+c_{4} t^{4}+c_{6}\left(t^{6}+t^{7}\right)+c_{8} t^{8}+c_{10}\left(t^{10}+t^{11}\right)+c_{12} t^{12}+c_{13} t^{13}+c_{14}\left(t^{14}+t^{15}\right)+\sum_{n \geq 16} c_{n} t^{n}
$$

We will now determine the $U_{j}$ of Definition 26 by computing their complements in the affine spaces $V_{j}$. Recall that the space $V_{\mathfrak{j}}$ depended on a choice of generators of $\mathfrak{j}$; we use the set of generators indicated (which is always the minimal set of generators). For $I$ an ideal of $\mathcal{O}$, we say its type is the semigroup ideal $\nu(I) \subset \Gamma$.

- The complement of $U_{i+(0,2,5)}$ inside $V_{i+(0,2,5)}$ will be the ideals whose type is $i+(0,2,5,7)$. As $i+9 \in \Gamma$ since $i+5 \in \Gamma$, the $i+7$ appears in the complement of a hyperplane if at all. Thus $U_{\Gamma}$ has Euler characteristic 1. On the other hand, we see in Corollary 31 that an ideal of type $i+(0,2,5)$ always has three generators. Thus

$$
\int_{U_{i+(0,2,5)}}\left(1-a^{2}\right)^{m-1} d \chi=\left(1-a^{2}\right)^{2}
$$

- The complement of $U_{i+(0,2,7)}$ in $V_{i+(0,2,7)}$ consists of semigroup ideals whose type is $i+$ $(0,2,7,9)$. Since $i+7$ is in the semigroup ideal, $i+9$ fails to be in the semigroup ideal either on a hyperplane or in all of $U_{i+(0,2,7)}$. Moreover when $i+9$ fails to be in the semigroup ideal, by Lemma 30 , the generators of degree $i, i+2$ generate the ideal. Thus

$$
\int_{U_{i+(0,2,5)}}\left(1-a^{2}\right)^{m-1} d \chi=\left(1-a^{2}\right)
$$


- $U_{i+(0,2,5,7)}=V_{i+(0,2,5,7)}$. Since $i+9$ is in the semigroup ideal, the space on which the generator of degree seven is not needed is the complement of a hyperplane and thus has Euler characteristic zero. Thus:

$$
\int_{U_{i+(0,2,5,7)}}\left(1-a^{2}\right)^{m-1} d \chi=\left(1-a^{2}\right)^{3}
$$

- $U_{i+(0,2,7,9)}=V_{i+(0,2,7,9)}$. Since 7 and 9 are both in the semigroup ideal, the generator of degree $i+7$ is unnecessary in the complement of a hyperplane, and the generator of degree $i+9$ is unnecessary in the complement of a parallel hyperplane.

$$
\int_{U_{i+(0,2,7,9)}}\left(1-a^{2}\right)^{m-1} d \chi=2\left(1-a^{2}\right)^{2}-\left(1-a^{2}\right)
$$

- The complement of $U_{i+(0,2,9)}$ in $V_{i+(0,2,9)}$ consists of the locus where the type is $(0,2,7,9)$ or $(0,2,9,11)$. We already understand that the first happens, if at all, on the the complement of a hyperplane. Let $U_{i+0,2,9}^{\prime}$ be the affine space on which the type is not $(0,2,7,9)$. Here the generators may be written:

$$
\begin{aligned}
f & =t^{i}+a t^{i+1}+a_{3} t^{i+3}+a_{5} t^{i+5}+a_{7} t^{i+7}+a_{11} t^{i+11} \\
g & =t^{i+2}+(a+1) t^{i+3}+b_{3} t^{i+5}+b_{5} t^{i+7}+b_{9} t^{i+11} \\
h & =t^{i+9}+c t^{i+11}
\end{aligned}
$$

Writing $x=t^{4}$ and $y=t^{6}+t^{7}$, we see that

$$
\left(x^{2}+x y(a+1)\right) f-y g+2 h=\left((a+1)^{2}+2 c+a_{3}-b_{3}\right) t^{11}+O\left(t^{12}\right)
$$

Now let $U_{i+(0,2,9)}^{\prime \prime}$ be the locus where the above coefficient of $t^{11}$ vanishes; since it is given as $c=\left(\left(b_{3}-a_{3}\right)+(a+1)^{2}\right) / 2$, it is isomorphic to affine space. Restricting to $U_{i+(0,2,9)}^{\prime \prime}$, one checks that the only term of degree 11 , modulo $t^{12}$, is

$\left(2 x-\left(a^{2}-a_{3}+b_{3}\right) y\right) g+\left(2\left(a x^{2}+a_{3} x y-y\right)+\left(a^{2}-a_{3}+b_{3}\right)\left(x^{2}+x y(a+1)\right)\right) f$

and the coefficient of $t^{11}$ is $\left.C\left(a, a_{3}, a_{5}, b_{3}, b_{5}\right)=2 b_{5}-2 a_{5}+P\left(a, a_{3}, b_{3}\right)\right)$, where $P$ is some polynomial. Evidently $U_{i+(0,2,9)}$ is the locus inside $U_{i+(0,2,9)}^{\prime \prime}$ where $C$ vanishes. If either $i+5$ or $i+7$ is in $\Gamma$, then $C$ vanishes on a hypersurface isomorphic to affine space. In fact, by inspection of $\Gamma$, one sees that whenever $i+(0,2,9,11) \subset \Gamma$, either $i+5$ or $i+7$ is in $\Gamma$. In the case $i+(0,2,9,11) \not \subset \Gamma$ and hence $i+11 \notin \Gamma$, then $C$ vanishes identically, $U_{i+(0,2,9)}=U_{i+(0,2,9)}^{\prime \prime}$, which we already knew was an affine space. In any event $U_{i+(0,2,9)}$ has Euler characteristic 1 . Finally by Lemma 30 the generator of degree $i+9$ is superfluous, so:

$$
\int_{U_{i+(0,2,9)}}\left(1-a^{2}\right)^{m-1} d \chi=\left(1-a^{2}\right)
$$

- The complement of $U_{i+(0,2,9,11)}$ inside $V_{i+(0,2,9,11)}$ is the locus where the semigroup type is $i+(0,2,7,9)$. We have seen that the $i+7$ appears in the complement of a hyperplane, if at all. Thus $U_{i+(0,2,9,11)}$ is isomorphic to affine space. We have also seen that the generator of degree $i+9$ is always superfluous; by the argument given for $(0,2,9)$, the generator of 
degree $i+11$ is superfluous in the complement of an affine space. Thus:

$$
\int_{U_{i+(0,2,9,11)}}\left(1-a^{2}\right)^{m-1} d \chi=\left(1-a^{2}\right)^{2}
$$

This completes the determination of the integrals appearing in Equation (14). Summing the contributions yields complete agreement with the HOMFLY polynomial.

\section{REFERENCES}

[AGV] V. Arnold, S. Gusein-Zade, A. Varchenko, Singularities of Differentiable Maps, Vol. I \& II, Birkhauser 1988.

[AK] A. Altman and S. Kleiman, em Compactifying the Picard Scheme, Advances in Mathematics 35, pp. 50-112 (1980).

[B] A. Beauville, Counting rational curves on K3 surfaces, Duke Math. J. 97.1, pp. 99-108 (1999).

[Br] J. Briançon, Description de Hilb ${ }^{n} \mathbb{C}\{x, y\}$, Invent. Math. 41, pp. 45-89 (1977).

$[\mathrm{BH}]$ W. Bruns and J. Herzog, Cohen-Macaulay rings: revised edition, Cambridge Studies in Advanced Mathematics 39, Cambridge University Press (1993).

[vB] J. M. von Buskirk, Positive knots have positive Conway polynomials in Lecture Notes in Mathematics 1144 pp. 146-159, Springer (1985).

[CDG] A. Campillo, F. Delgado, and S. M. Gusein-Zade, The Alexander polynomial of a plane curve singularity via the ring of functions on it, Duke Math J. 118.1, pp. 125-156 (2003).

[CDG2] A. Campillo, F. Delgado, S. M. Gusein-Zade, Integrals with respect to the Euler characteristic over spaces of functions and the Alexander polynomial. Proc. Steklov Inst. Math. 3.238, pp. 134147 (2002)

[E] D. Eisenbud, Homological algebra on a complete intersection, with an application to group representations, Trans. AMS (1980).

[EN] D. Eisenbud and W. Neumann, Three-dimensional link theory and invariants of plane curve singularities, Ann. of Math. Studies 110, Princeton, 1985.

[G] A. Grothendieck, Techniques de construction et théorèms d'existence en géométrie algébraique. IV. Les schémas de Hilbert, Séminare Bourbaki, Vol. 6, Exp. 221, Soc. Math. France, Paris, pp. 249-276 (1995).

[GV] R. Gopakumar and C. Vafa, M-theory and topological strings II, hep-th/9812127.

[HOMFLY] P. Freyd, D. Yetter, J. Hoste, W. B. R. Lickorish, K. Millett, and A. Ocneanu, A new polynomial invariant of knots and links, Bull. Amer. Math. Soc. 12, p. 239 (2002).

[H] R. Hartshorne, Generalized divisors on Gorenstein curves and a theorem of Noether, J. Math. Kyoto Univ 26.3, pp. 375-386 (1986).

[I] A. Iarrobino. Punctual Hilbert schemes. Mem. AMS 188, 1977.

[J] V. F. R. Jones, Hecke algebra representations of braid groups and link polynomials, Ann. Math 126, pp. 335-388 (1987).

[Ka] A. Kawauchi, A survey of knot theory, Birkhauser 1996.

[KR] M. Khovanov, L. Rozansky, Matrix factorizations and link homology, I. [math.QA/0401268] and II. [math.QA/0505056].

[M] J. Milnor, Singular points of complex hypersurfaces, Princeton University Press 1968.

[ORS] A. Oblomkov, J. Rasmussen, and V. Shende, The Hilbert scheme of a singular curve and the HOMFLY homology of its link, to appear.

[Pi] J. Piontkowski, Topology of the compactified Jacobians of singular curves, Mathematische Zeitschrift bf 255.1, pp. 195-226 (2007)

[P] R. Pandharipande, Hilbert schemes of singular curves, web notes.

[PT3] R. Pandharipande and R. Thomas, Stable pairs and BPS invariants [arXiv:0711.3899].

[S] V. Shende, Hilbert schemes of points on a locally planar curve and the Severi strata of its versal deformation, [arXiv:1009.0914].

[W] E. Witten, Quantum field theory and the Jones polynomial, Comm. Math. Phys. 121, p. 351 (1989).

[Z2] O. Zariski, Studies in Equisingularity. II. Equisingularity in codimension 1 (and characteristic zero), Amer. J. Math. 87, pp. 972-1006 (1965).

[ZT] O. Zariski and B. Teissier, Le problème des modules pour les branches planes, Hermann, Paris, 1986. 


\begin{tabular}{|c|c|c|}
\hline$\Delta \in \operatorname{Mod}(\Gamma)$ & $8-\# \mathbb{N} \backslash \Delta$ & $i \mid i+\Delta \subset \Gamma$ \\
\hline$(0)$ & 0 & $0,4,6,8,10,12,13,14,16+$ \\
\hline$(0,1)$ & 6 & $12,13,16+$ \\
\hline$(0,3)$ & 5 & $13,14,16+$ \\
\hline$(0,5)$ & 4 & $8, \quad 12,13,14,16+$ \\
\hline$(0,7)$ & 3 & $6, \quad 10,12,13,14,16+$ \\
\hline$(0,9)$ & 2 & $4, \quad 8,10,12,13,14,16+$ \\
\hline$(0,11)$ & 2 & $6,8,10,12,13,14,16+$ \\
\hline$(0,15)$ & 1 & $4,6,8,10,12,13,14,16+$ \\
\hline$(0,2)$ & 2 & $4,6,8,10,12, \quad 14,16+$ \\
\hline$(0,1,3)$ & 7 & $13,16+$ \\
\hline$(0,3,5)$ & 6 & $13,14,16+$ \\
\hline$(0,5,7)$ & 5 & $12,13,14,16+$ \\
\hline$(0,7,9)$ & 4 & $10,12,13,14,16+$ \\
\hline$(0,9,11)$ & 3 & $8,10,12,13,14,16+$ \\
\hline$(0,1,2)$ & 7 & $12, \quad 16+$ \\
\hline$(0,2,3)$ & 6 & $14,16+$ \\
\hline$(0,2,5)$ & 5 & $8, \quad 12$ \\
\hline$(0,2,7)$ & 4 & $6, \quad 10,12$ \\
\hline$(0,2,9)$ & 3 & $4, \quad 8,10,12$ \\
\hline$(0,2,11)$ & 3 & $6,8,10,12$ \\
\hline$(0,1,2,3)$ & 8 & $16+$ \\
\hline$(0,2,3,5)$ & 7 & $14,16+$ \\
\hline$(0,2,5,7)$ & 6 & $14,16+$ \\
\hline$(0,2,7,9)$ & 5 & $14,16+$ \\
\hline$(0,2,9,11)$ & 4 & $8,10,12$ \\
\hline
\end{tabular}

FIGURE 1. The combinatorial data required by Equation (14).

Alexei Oblomkov

Department of Mathematics

University of Massachusetts, Amherst

Amherst, MA 01003

oblomkov@math.umass.edu

Vivek Shende

Department of Mathematics

Massachusetts Institute of Technology

Cambridge, MA 02139

vivek@math.mit.edu 